\title{
EXCISION FOR SIMPLICIAL SHEAVES ON THE STEIN SITE AND GROMOV'S OKA PRINCIPLE
}

\author{
FINNUR LÁRUSSON \\ University of Western Ontario
}

\begin{abstract}
A complex manifold $X$ is said to satisfy the Oka-Grauert property if the inclusion $\mathcal{O}(S, X) \hookrightarrow \mathcal{C}(S, X)$ is a weak equivalence for every Stein manifold $S$, where the spaces of holomorphic and continuous maps from $S$ to $X$ are given the compact-open topology. Gromov's Oka principle states that if $X$ has a spray, then it has the Oka-Grauert property. The purpose of this paper is to investigate the Oka-Grauert property using homotopical algebra. We embed the category of complex manifolds into the model category of simplicial sheaves on the site of Stein manifolds. Our main result is that the Oka-Grauert property is equivalent to $X$ representing a finite homotopy sheaf on the Stein site. This expresses the Oka-Grauert property in purely holomorphic terms, without reference to continuous maps.
\end{abstract}

\section{Introduction}

The purpose of this paper is to investigate Gromov's Oka principle using abstract homotopy theory and to recast it in intrinsic, holomorphic terms, and thereby to introduce some of the concepts and methods of homotopical algebra into complex geometry.

The Oka principle is a vague maxim, supported by many results. It may be phrased by saying that on a Stein manifold (complex submanifold of Euclidean space), analytic problems of a cohomological nature have only topological obstructions. It has a long and venerable history, starting with the 1939 result of Oka stating, in modern language, that a holomorphic line bundle on a domain of holomorphy is trivial if it is topologically trivial. Deep generalizations to vector bundles and certain other fiber bundles were obtained by Grauert in the late 1950s. Another manifestation of the Oka principle is the 1975 result of Cornalba and Griffiths that every rational cohomology class of degree $2 k$ on a Stein

1991 Mathematics Subject Classification. Primary: 32Q28; secondary: 18F10, 18F20, 18G30, 18G55, 32E10, 32H02, 55U35.

The author was supported in part by the Natural Sciences and Engineering Research Council of Canada.

Completed December 2000; minor changes April 2002.

Typeset by $\mathcal{A M}_{\mathcal{M}}$-TEX 
manifold is a rational multiple of the fundamental class of a $k$-codimensional analytic subvariety. For a survey, see [19].

A major development appeared in Gromov's 1989 paper [13]. He discovered that if a complex manifold $X$ has a geometric structure called a spray, then $X$ satisfies what we shall call the Oka-Grauert property, meaning that the inclusion $\mathcal{O}(S, X) \hookrightarrow \mathcal{C}(S, X)$ is a weak equivalence for every Stein manifold $S$, where the spaces of holomorphic and continuous maps from $S$ to $X$ are given the compact-open topology. In particular, every continuous map from a Stein manifold to $X$ can be deformed to a holomorphic map. A spray on $X$ consists of holomorphic maps $\mathbb{C}^{m} \rightarrow X, 0 \mapsto x$, one for each $x \in X$, submersive at 0 , and varying holomorphically with $x$. For a detailed proof of Gromov's theorem and a thorough introduction, see [8]. A more general version for sections of submersions is contained in [10]. Gromov's Oka principle has been applied to the famous problem of embedding Stein manifolds into Euclidean spaces of the smallest possible dimension: Schürmann has used it to prove Forster's conjecture in higher dimensions [28], following work of Eliashberg and Gromov [3]. Other applications and variants may be found in $[4,5,6,7,9]$.

The Oka-Grauert property certainly has a homotopy-theoretic flavour. Our goal is to turn this impression into a precise statement in an abstract homotopy-theoretic setting. At the same time, we will express the Oka-Grauert property in purely holomorphic terms, without reference to continuous maps.

Abstract homotopy theory, also known as homotopical algebra, is due to Quillen [2, $12,14,16,27]$. Its fundamental notion is that of a model category: a category satisfying certain axioms that allow us to develop an analogue of ordinary homotopy theory. There has been much activity in recent years in both the theory and applications of homotopical algebra, the most notable example being the development of motivic homotopy theory, i.e., the homotopy theory of schemes, leading to Voevodsky's proof of the Milnor conjecture $[31,32]$. As far as I know, the present paper is the first attempt to introduce homotopical algebra into analytic geometry.

The first step, just as for schemes, is to embed the category of complex manifolds into the model category of simplicial objects in a topos by a Yoneda embedding of some sort, where we can then do homotopy theory with them. Here, this is done in Section 4. Whereas in algebraic geometry the focus is on generalized cohomology theories and ultimately motives, on the analytic side it seems of more immediate interest to try to do algebraic topology with complex manifolds and holomorphic maps instead of topological spaces and continuous maps, and then our attention is immediately drawn to Gromov's Oka principle. For the purpose of studying the Oka principle, we associate to a complex manifold $X$ the simplicial sheaf $s \mathcal{O}(\cdot, X)$ on the site of all Stein manifolds. Here, spaces of holomorphic maps are given the compact-open topology; it is for technical reasons that we turn them into simplicial sets by applying the singular functor $s$. Using the compactopen topology allows us to work at a relatively simple technical level: we neither have to localize nor stabilize to get something interesting. 
Among other things, a new notion of weak equivalence between complex manifolds emerges, weaker than biholomorphism and stronger than topological weak equivalence (it can tell apart the punctured plane and the punctured disc). The definition is simple - a holomorphic map $X \rightarrow Y$ is a weak equivalence if it induces a topological weak equivalence $\mathcal{O}(S, X) \rightarrow \mathcal{O}(S, Y)$ for every Stein manifold $S$ - but the point is that it fits into a model structure.

The main result of this paper is Theorem 2.1, later rephrased as Theorem 4.3. It states that a complex manifold $X$ has the Oka-Grauert property if and only if the simplicial sheaf $s \mathcal{O}(\cdot, X)$ is a finite homotopy sheaf on the Stein site. This homotopy-theoretic property is also called finite excision. It gives rise to Mayer-Vietoris sequences of homotopy groups and is familiar from topology and appears nowadays in algebraic geometry: see e.g. [25, $\S 3.1 .2]$. I have tried to make the proof of Theorem 2.1 as understandable as possible to those unfamiliar with homotopy theory. However, the very definition of excision requires the notion of a homotopy limit (a deformation invariant approximate limit), for which I refer the reader to [12, VIII.2] and [14, Ch. 19]. The proof uses the main result of Section 3, Theorem 3.4, whose crucial ingredient is a classical theorem of Brown and Gersten [1, Thm. 1], foundational in the homotopy theory of simplicial sheaves. Section 3 is pure homotopy theory and constitutes the bulk of the paper. The proof of Theorem 2.1 also uses Siu's theorem on the existence of Stein neighbourhoods of Stein subvarieties, and Whitney's lemma on decomposing an open subset in Euclidean space into a union of cubes with special properties.

Those familiar with the homotopy theory of simplicial sheaves would now ask if the Oka-Grauert property is actually equivalent to descent, making Gromov's Oka principle somewhat analogous to such results as Brown-Gersten, Nisnevich, and Thomason descent in algebraic geometry [24]. Descent may be loosely described as a homotopic localto-global property, stronger than excision. I do not know the answer: the finiteness properties that make descent possible in algebra do not hold in analysis. I hope to address this question in future work. In the meantime, Section 5 contains a partial descent theorem of sorts for quasi-projective manifolds.

It should be emphasized that this paper is not about the proof of Gromov's theorem at all. The ideas presented in the paper may shed new light on the proof, but this possibility is not pursued here. Rather, the paper is about the Oka-Grauert property itself and its homotopy-theoretic meaning in a new model-categorical context for complex manifolds.

A word about terminology: we take manifolds to be second countable by definition, but not necessarily connected.

Acknowledgements. I am grateful for discussions with Paul Balmer, Dan Christensen, Gaunce Lewis, Sergei Yagunov, and especially Rick Jardine, who has generously and patiently answered many questions of mine. 


\section{The Oka-Grauert property is equivalent to finite excision}

We say that a complex manifold $X$ (second countable but not necessarily connected) has the Oka-Grauert property if the inclusion map

$$
\mathcal{O}(S, X) \hookrightarrow \mathcal{C}(S, X)
$$

is a weak equivalence for all Stein manifolds $S$, where the spaces of holomorphic and continuous maps from $S$ to $X$ carry the compact-open topology. This means that the inclusion induces isomorphisms of all homotopy groups for all base points, as well as a bijection of path components. In particular, surjectivity on path components means that every continuous map $S \rightarrow X$ is homotopic to a holomorphic map. Note that requiring $S$ to be connected results in an equivalent condition.

We say that $X$ satisfies finite excision if whenever $\left\{U_{1}, \ldots, U_{m}\right\}$ is a finite cover of a Stein manifold $S$ by Stein open subsets, $\mathcal{O}(S, X)$ is not only the limit but also the homotopy limit of the diagram whose objects are the spaces $\mathcal{O}\left(U_{i_{1}} \cap \cdots \cap U_{i_{k}}, X\right)$ for $1 \leq i_{1}<\cdots<i_{k} \leq m$ and $k=1, \ldots, m$, and whose arrows are the restriction maps. This diagram forms an $m$-dimensional cube with one vertex missing; the limit or homotopy limit provides the missing vertex. For $m=2$, this simply means that the square

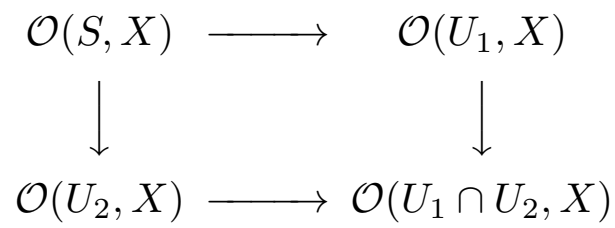

is not only a pullback but also a homotopy pullback.

Let us make clear what we mean by a homotopy limit. We view homotopy limits as determined only up to weak equivalence. We say that a topological space $Y$ with a map to a diagram $\mathcal{X}$ of spaces over an index category $J$ is the homotopy limit of $\mathcal{X}$ (or more properly that the diagram $Y \rightarrow \mathcal{X}$ is a homotopy limit) if and only if the singular set $s Y$ of $Y$ is the homotopy limit of the diagram $s \mathcal{X}$ of simplicial sets. This in turn means that if $\mathcal{A}$ is a fibrant model for $s \mathcal{X}$ in the category of diagrams of simplicial sets over $J$ with the pointwise cofibration structure, then the natural map from $s Y$ to the limit of $\mathcal{A}$ is a weak equivalence [12, VIII.2.11]. It is my understanding that this definition is standard (up to weak equivalence): it agrees up to weak equivalence with the definitions of Bousfield-Kan and Hirschhorn [14, Ch. 19]. The homotopy limit of a diagram of spaces or simplicial sets may also be described somewhat explicitly as the function space or complex of morphisms to the diagram from a certain diagram associated to the index category [12, VIII.2.3; 14, 19.1.10]. We will make frequent use of the basic fact that if one of two weakly equivalent diagrams of spaces or fibrant simplicial sets is a homotopy limit, then so is the other one.

Our main result is that the Oka-Grauert property is equivalent to finite excision. 
2.1. Theorem. A complex manifold has the Oka-Grauert property if and only if it satisfies finite excision.

Proof. Let $X$ be a complex manifold with the Oka-Grauert property. Consider a finite cover of a Stein manifold $S$ by Stein open subsets $U_{1}, \ldots, U_{m}$. We have two $m$-dimensional cube diagrams of spaces, one with objects $\mathcal{O}\left(U_{i_{1}} \cap \cdots \cap U_{i_{k}}, X\right)$, and the other with objects $\mathcal{C}\left(U_{i_{1}} \cap \cdots \cap U_{i_{k}}, X\right)$ for $1 \leq i_{1}<\cdots<i_{k} \leq m$. We have a morphism of inclusions from the first diagram to the second one, consisting of weak equivalences by assumption (here we need to know that the intersection of Stein open sets is Stein; see Lemma 4.1). Hence, the induced map between the homotopy limits of the two diagrams is a weak equivalence [12, VIII.2.2]. By Theorem 3.4 below, the homotopy limit of the second diagram is $\mathcal{C}(S, X)$, which by assumption is weakly equivalent to $\mathcal{O}(S, X)$.

Conversely, assume $X$ is a complex manifold satisfying finite excision and let $S$ be a Stein manifold. We first reduce our problem to the case when $S$ is a domain in Euclidean space. As noted above, we may take $S$ to be connected, so $S$ embeds into some Euclidean space. Then, by a theorem of Siu [29, Cor. 1], there is a connected Stein neighbourhood $V$ of $S$ and a holomorphic retraction $\rho: V \rightarrow S$. Let $\iota: S \hookrightarrow V$ be the inclusion, so $\rho \circ \iota=\operatorname{id}_{S}$, and we have a diagram

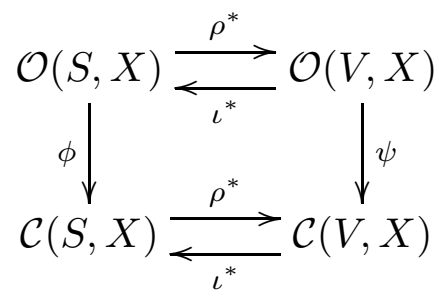

where $\phi$ and $\psi$ are the inclusions. Now suppose $\psi$ is a weak equivalence. Since $\iota^{*} \circ \rho^{*}=\mathrm{id}$, $\rho^{*}$ induces monomorphisms and $\iota^{*}$ induces epimorphisms on all homotopy groups. Hence, $\rho^{*} \circ \phi=\psi \circ \rho^{*}$ induces monomorphisms on all homotopy groups so $\phi$ does too, and $\phi \circ \iota^{*}=\iota^{*} \circ \psi$ induces epimorphisms on all homotopy groups so $\phi$ does too, and $\phi$ is a weak equivalence.

To complete the proof we need to show that $\mathcal{O}(V, X) \hookrightarrow \mathcal{C}(V, X)$ is a weak equivalence when $V$ is a Stein domain in Euclidean space. We want to express $V$ as a finite union of open subsets all of whose connected components are convex. This can surely be done in many ways. We shall refer to Whitney's classical lemma on decomposing an open set in Euclidean space into a union of cubes with special properties [30, VI.1]. We get that $V=U_{1} \cup \cdots \cup U_{m}$, where each $U_{i}$ is a disjoint union of open cubes with sides parallel to the coordinate axes. (This is not stated explicitly in [30], but may easily be obtained from there.) Then every intersection $U=U_{i_{1}} \cap \cdots \cap U_{i_{k}}$ is a disjoint union of open boxes and hence Stein. Each box is holomorphically contractible in the sense that the identity map can be joined to a constant map by a continuous family of holomorphic maps, so the inclusion $\mathcal{O}(U, X) \hookrightarrow \mathcal{C}(U, X)$ is clearly a weak equivalence. Now we look 
at two $m$-dimensional cube diagrams of spaces, one with objects $\mathcal{O}\left(U_{i_{1}} \cap \cdots \cap U_{i_{k}}, X\right)$ and the other with objects $\mathcal{C}\left(U_{i_{1}} \cap \cdots \cap U_{i_{k}}, X\right)$ for $1 \leq i_{1}<\cdots<i_{k} \leq m$. As above, we see that the induced map between the homotopy limits of the two diagrams is a weak equivalence. Since $X$ satisfies finite excision, and by Theorem 3.4, this map is the inclusion $\mathcal{O}(V, X) \hookrightarrow \mathcal{C}(V, X)$ (at least up to weak equivalence), and the proof is complete.

The proof shows that a complex manifold $X$ satisfies finite excision if and only if $\mathcal{O}(S, X) \rightarrow \mathcal{O}(\mathcal{B}, X)$ is a homotopy limit for every Stein basis $\mathcal{B}$ for a binoetherian subtopology on a Stein manifold $S$, viewed as a subdiagram of the site of $S$.

The proof also shows that the Oka-Grauert property for a complex manifold $X$ is equivalent to the inclusion $\mathcal{O}(V, X) \hookrightarrow \mathcal{C}(V, X)$ being a weak equivalence for all domains of holomorphy $V$ in $\mathbb{C}^{n}$ for all $n \geq 1$. To some extent it is therefore a matter of taste whether one chooses to work with Stein manifolds or domains of holomorphy in Euclidean space in the present context.

If $X$ has the Oka-Grauert property and $S$ is a Stein manifold, it is natural to ask whether the weak equivalence $\mathcal{O}(S, X) \hookrightarrow \mathcal{C}(S, X)$ is actually a homotopy equivalence. When $S$ is algebraic, a little topology shows that the inclusion has a right homotopy inverse, so there is a continuous way of associating to each continuous map $S \rightarrow X$ a holomorphic map homotopic to it. Note that we do not assert that the homotopy inverse fixes holomorphic maps.

2.2. Theorem. Let $S$ be an affine algebraic manifold, i.e., a Stein manifold biholomorphic to an algebraic submanifold of Euclidean space. If $X$ is a complex manifold and the inclusion $\mathcal{O}(S, X) \hookrightarrow \mathcal{C}(S, X)$ is a weak equivalence, then it has a right homotopy inverse.

Proof. Being a smooth manifold, $X$ has a countable triangulation [26, 10.6], so $X$ is homeomorphic to a countable $\mathrm{CW}$ complex. Also, $S$ is homotopy equivalent to a finite CW complex $K$ [23, Lemma A.3], and $\mathcal{C}(S, X)$ is homotopy equivalent to $\mathcal{C}(K, X)$, which has the homotopy type of a (countable) $\mathrm{CW}$ complex [11, 5.2.5], so $\mathcal{C}(S, X)$ has the homotopy type of a CW complex. Hence, the natural map $a:|s \mathcal{O}(S, X)| \rightarrow|s \mathcal{C}(S, X)| \rightarrow$ $\mathcal{C}(S, X)$, which is a weak equivalence by assumption, has a homotopy inverse $b$. Let $c$ be the natural map $|s \mathcal{O}(S, X)| \rightarrow \mathcal{O}(S, X)$. By adjunction, $a=i c$, where $i$ is the inclusion $\mathcal{O}(S, X) \hookrightarrow \mathcal{C}(S, X)$. Now $i(c b)=a b$ is homotopic to the identity on $\mathcal{C}(S, X)$, so $c b$ is a right homotopy inverse for $i$.

\section{Excision and Brown-Gersten descent}

The main purpose of this section is to establish the excision property of $s \mathcal{C}(\cdot, X)$ used in the previous section (Theorem 3.4). One feels that it should be possible to verify this directly using the explicit description of the homotopy limit given in [12, VIII.2.3] and $[14,19.1 .10]$, but rather than attempt this, we give a proof based on Brown-Gersten descent (Theorem 3.1). Brown-Gersten descent is surely well known among experts, but 
in the absence of a good reference, we have provided a detailed proof. Theorems 3.3 and 3.4 are new as far as I know. We start with a brief review of the basic notions of the homotopy theory of simplicial presheaves.

Let $\mathcal{S}$ be a small Grothendieck site. A simplicial presheaf on $\mathcal{S}$ is a contravariant functor from $\mathcal{S}$ to the category $s$ Set of simplicial sets. There is a standard model structure on the category $s$ Pre $\mathcal{S}$ of simplicial presheaves on $\mathcal{S}$ in which the cofibrations are monomorphisms, i.e., pointwise injections (where pointwise means at every object of the site), and a weak equivalence is a map that induces isomorphisms of all homotopy sheaves $[17$, p. 59]. If $\mathcal{S}$ has enough points, e.g. if it is the site of a topological space, then this is equivalent to the map inducing weak equivalences of all stalks. A weak equivalence is still a weak equivalence with respect to any finer topology. Fibrations are defined by a right lifting property. There is an induced model structure on the full subcategory $s$ Shv $\mathcal{S}$ of simplicial sheaves on $\mathcal{S}$. More is true: both categories are proper, simplicial model categories [17, 18]. There is another model structure on $s$ Pre $\mathcal{S}$ given by the trivial topology on $\mathcal{S}$, in which the only covers are those consisting of a single isomorphism. In this structure, the weak equivalences are the pointwise weak equivalences. The words fine and finely shall refer to the former model structure, and the words coarse and coarsely to the latter. A map of simplicial presheaves which is a weak equivalence will be referred to as acyclic. (We are trying not to overuse the word trivial.) A coarse weak equivalence is a fine weak equivalence; a fine fibration is a coarse fibration.

The concept of a fibrant object is fundamental in homotopy theory. We will now define several important weaker notions and briefly describe their relationships.

We say that a simplicial presheaf $G$ on $\mathcal{S}$ satisfies descent if any fine weak equivalence from $G$ to a finely fibrant simplicial presheaf on $\mathcal{S}$ is coarsely acyclic. Equivalently (using the Whitehead Theorem that a weak equivalence between bifibrant objects is a homotopy equivalence), a finely fibrant model for $G$ is also a coarsely fibrant model for $G$. This notion is invariant under coarse weak equivalences. A finely fibrant simplicial presheaf satisfies descent.

It may be shown that a coarsely fibrant simplicial presheaf $G$ on $\mathcal{S}$ is both pointwise fibrant and flabby (or flasque), which means that the restriction map $G(U) \rightarrow G(V)$ is a fibration for every monomorphism $V \rightarrow U$ in $\mathcal{S}$.

Now let $X$ be a topological space. We say that a pointwise fibrant simplicial presheaf $G$ on $X$, i.e., a presheaf of Kan complexes, satisfies excision (or two-set excision) if $G(\varnothing)$ is contractible (this is true if $G$ is a sheaf) and whenever $U$ and $V$ are open in $X$, the square

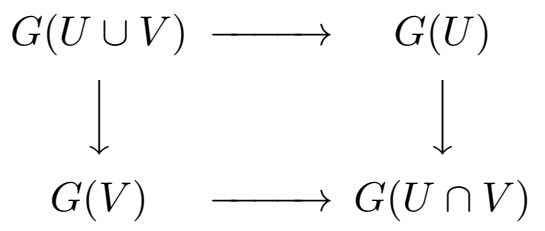

is a homotopy pullback. This notion was introduced by Brown and Gersten [1, $§ 2]$, who 
used the term pseudo-flasque. It is clearly invariant under coarse weak equivalences. If $G$ is a flabby simplicial sheaf (so $G$ is in particular pointwise fibrant), then $G$ satisfies excision: the diagram $G(U) \rightarrow G(U \cap V) \leftarrow G(V)$ is fibrant in its diagram category, so the ordinary pullback $G(U \cup V)$ is the homotopy pullback [12, VI.1.8]. If $G$ is a pointwise fibrant simplicial presheaf satisfying descent, consider the fine weak equivalence from $G$ to its sheafification $a G$, and the fine weak equivalence from $a G$ to a finely fibrant model $F$ in $s$ Shv $X$. Then $F$ is also finely fibrant in $s \operatorname{Pre} X$, so $F$ is flabby and satisfies excision, and since $F$ and $G$ are finely and hence coarsely equivalent, $G$ does too. Under a strong finiteness condition on $X$, the converse holds: this is Brown-Gersten descent. We say that a topological space is binoetherian if both the open sets and the irreducible closed sets satisfy the ascending chain condition. An important example is a space with a finite topology.

3.1. Theorem (Brown-Gersten descent). For pointwise fibrant simplicial presheaves on a binoetherian space, excision is equivalent to descent.

Proof. Let $G$ be a pointwise fibrant simplicial presheaf on a binoetherian space, and suppose that $G$ satisfies excision. Let $G \rightarrow F$ be a fine weak equivalence from $G$ to a finely fibrant simplicial presheaf $F$. Then $F$ satisfies descent and hence excision. The theorem now follows from the next result.

3.2. Proposition. Let $F$ and $G$ be pointwise fibrant simplicial presheaves satisfying excision on a binoetherian space $X$. Then a fine weak equivalence $G \rightarrow F$ is coarsely acyclic.

Our argument is an adaptation and explication of Morel and Voevodsky's proof of unstable Nisnevich descent in [25, §3.1.2].

Proof. Let $F^{\prime}, G^{\prime}$ be coarsely fibrant models in $s$ Pre $X$ for $F, G$ respectively. Factor the induced map $G^{\prime} \rightarrow F^{\prime}$ as a coarse weak equivalence $G^{\prime} \rightarrow G^{\prime \prime}$ followed by a coarse fibration $G^{\prime \prime} \rightarrow F^{\prime}$. Then $G^{\prime \prime}$ is coarsely fibrant and it suffices to show that the map $G^{\prime \prime} \rightarrow F^{\prime}$ is coarsely acyclic. By replacing $F, G$ by $F^{\prime}, G^{\prime \prime}$, we may assume that $F$ and $G$ are coarsely fibrant and hence flabby and pointwise fibrant, and that the fine weak equivalence $G \rightarrow F$ is a coarse fibration and hence a pointwise fibration.

It suffices to show that for any open set $U$ in $X$ and any vertex $x$ in $F(U)$, the fibre of the fibration $G(U) \rightarrow F(U)$ over $x$ is contractible (in particular nonempty): then $G(U) \rightarrow F(U)$ is a weak equivalence (if $F(U)$ is empty, then so is $G(U)$ and this is still true). Note that $U$ is binoetherian in the subspace topology, so we may assume that $U=X$. Fix a vertex $x$ in $F(X)$ and consider the simplicial presheaf $K$ on $X$ that associates to an open set $V$ in $X$ the fibre of $G(V) \rightarrow F(V)$ over the image of $x$ in $F(V)$. Note that $K$ is a pullback of a diagram $G \rightarrow F \leftarrow *$ of simplicial presheaves on $X$, where * denotes the final simplicial presheaf. Hence, $K$ is coarsely fibrant, and therefore flabby and pointwise fibrant. 
We need to show that $K$ is pointwise contractible. Since $K$ is stalkwise contractible (pullbacks commute with filtered colimits, so taking fibres commutes with taking stalks), this follows from [1, Thm. 1] along with the remark at the end of [1, 2$]$, once we know that $K$ satisfies excision. To complete the proof, let us verify this.

First of all, since $F(\varnothing)$ and $G(\varnothing)$ are contractible, so is the fibre $K(\varnothing)$. Now let $U$ and $V$ be open in $X$. Since $K$ is flabby and pointwise fibrant, the homotopy pullback of $K(U) \rightarrow K(U \cap V) \leftarrow K(V)$ is the ordinary pullback, so we need to show that the natural map $K(U \cup V) \rightarrow K(U) \times_{K(U \cap V)} K(V)$ is a weak equivalence. By the cogluing lemma [12, II.8.13] applied to the natural map from the pullback square

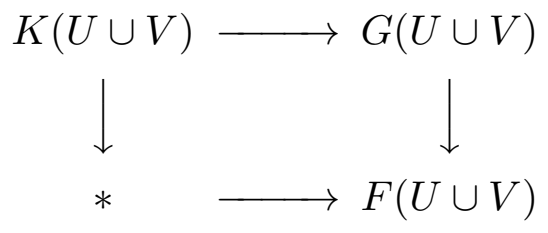

to the pullback square

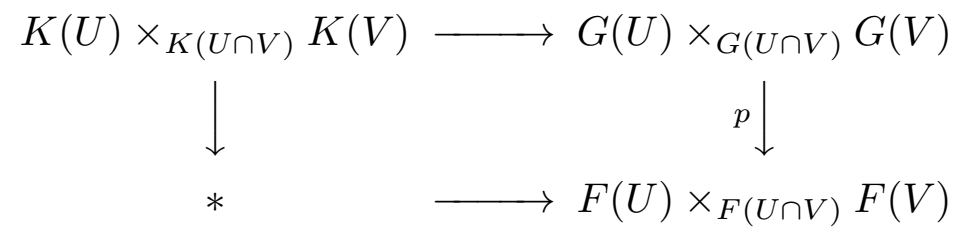

we would be done if $p$ was a fibration. Since this is not to be expected, we need to replace $p$ by a fibration in a reasonable way.

We shall work in the category of squares of simplicial sets of the type

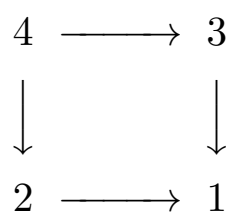

This category carries the pointwise cofibration and the pointwise fibration simplicial model structures [12, p. 403]. In both of them, the weak equivalences are the pointwise weak equivalences. We shall write $Q_{F}$ for the square

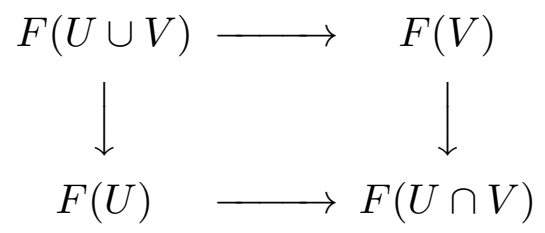

and similarly for $G$ and $K$. In the pointwise cofibration structure, factor $Q_{G} \rightarrow Q_{F}$ as a weak equivalence $Q_{G} \rightarrow Q$ followed by a fibration $Q \rightarrow Q_{F}$. Using the fibration $Q \rightarrow Q_{F}$, 
one can show that $Q$ is pointwise fibrant and the maps $Q_{2}, Q_{3} \rightarrow Q_{1}$ are fibrations. Since $Q_{G}$ is a homotopy pullback, so is $Q$, and the natural map $Q_{4} \rightarrow Q_{2} \times_{Q_{1}} Q_{3}$ is a weak equivalence.

The cogluing lemma applied to the natural map from the pullback square

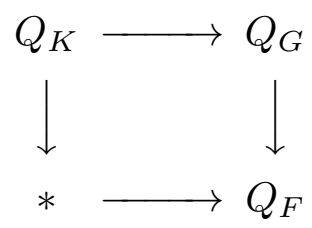

to the pullback square

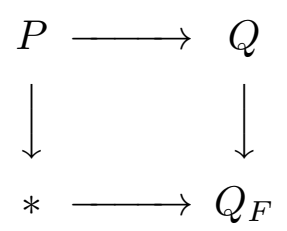

with respect to the pointwise fibration structure (which is right proper) shows that $Q_{K} \rightarrow$ $P$ is a weak equivalence (we invoke the fact that a fibration in the pointwise cofibration structure is a pointwise fibration). Also, $P$ is fibrant in the pointwise cofibration structure. Hence, it suffices to show that the square $P$ is a homotopy pullback, which is the case if and only if the natural map $P_{4} \rightarrow P_{2} \times{ }_{P_{1}} P_{3}$ is a weak equivalence.

By the cogluing lemma applied to the natural map from the pullback square

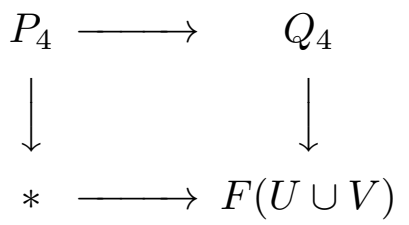

to the pullback square

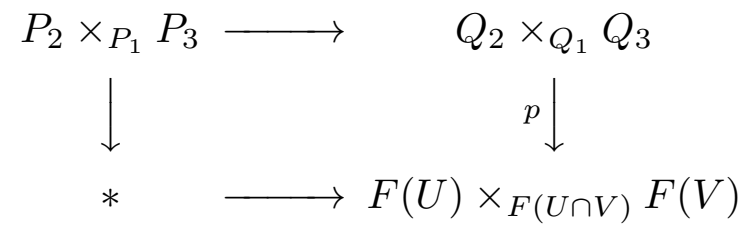

it suffices to show that the map $p$ is a fibration. Therefore, to complete the proof, we need to show that if $R \rightarrow S$ is a fibration of squares in the pointwise cofibration structure, then $R_{2} \times{ }_{R_{1}} R_{3} \rightarrow S_{2} \times S_{1} S_{3}$ is a fibration of simplicial sets. (Having a pointwise fibration is not enough.) A square

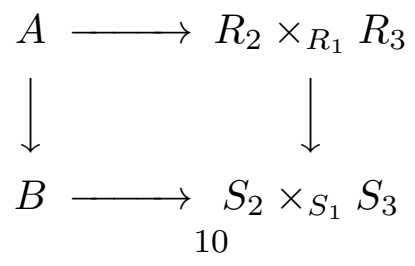


is the same thing as a square

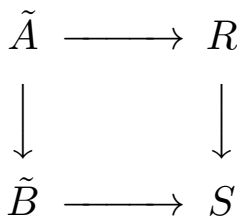

of squares, where $\tilde{A}$ is the square

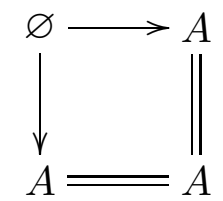

and $\tilde{B}$ is defined similarly. A map $A \rightarrow B$ is an acyclic cofibration if and only if the induced map $\tilde{A} \rightarrow \tilde{B}$ is a pointwise acyclic cofibration, and then a lifting in the latter square gives a lifting in the former.

Using Brown-Gersten descent, we can now strengthen the excision condition.

3.3. Theorem. Let $G$ be a pointwise fibrant simplicial presheaf satisfying excision on a topological space $X$. Let $\mathcal{B}$ be a basis for a binoetherian subtopology on an open set $U$ in $X$, viewed as a subdiagram of the site of $X$. Then $G(U)$ is the homotopy limit of $G \mid \mathcal{B}$.

Recall that a collection $\mathcal{B}$ of open subsets in a topological space is a basis for a subtopology on $\bigcup \mathcal{B}$ if whenever $U, V \in \mathcal{B}$ and $p \in U \cap V$, there is $W \in \mathcal{B}$ with $p \in W \subset U \cap V$. We do not assume that a basis is closed under intersections. The definition of excision refers to the case when $\mathcal{B}$ consists of two open sets in $X$ and their intersection.

Proof. We may assume that $X=U$ is binoetherian and $\mathcal{B}$ is a basis for the topology on $X$. By Brown-Gersten descent, $G$ satisfies descent, so there is a coarse weak equivalence from $G$ to a finely fibrant simplicial sheaf $F$ on $X$. Let us show that $F \mid \mathcal{B}$ is fibrant in the diagram category $s$ Set $^{\mathcal{B}^{\text {op }}}$ with the pointwise cofibration structure. Let $A \rightarrow B$ be an acyclic cofibration in $s$ Set $^{\mathcal{B}^{\text {op }}}$ and $A \rightarrow F \mid \mathcal{B}$ be a morphism. Now $A$ and $B$ yield étale spaces over $X$ with sheaves of sections $\tilde{A}$ and $\tilde{B}$ respectively, such that the induced map $\tilde{A} \rightarrow \tilde{B}$ is a finely acyclic cofibration in $s$ Pre $X$. Of course the diagram $F \mid \mathcal{B}$ yields the sheaf $F$ itself in this way, so the map $A \rightarrow F \mid \mathcal{B}$ factors through $\tilde{A} \mid \mathcal{B}$. Since $F$ is finely fibrant, $\tilde{A} \rightarrow F$ factors through $\tilde{B}$, so $A \rightarrow F \mid \mathcal{B}$ factors through $B$. This shows that $F \mid \mathcal{B}$ is a fibrant model for $G \mid \mathcal{B}$ in $s \operatorname{Set}^{\mathcal{B}^{\text {op }}}$. Hence, the homotopy limit of $G \mid \mathcal{B}$ is weakly equivalent to the limit of $F \mid \mathcal{B}$, which is $F(X)$ since $F$ is a sheaf, and $F(X)$ is weakly equivalent to $G(X)$.

The following theorem gives the excision property of $\mathcal{C}(\cdot, X)$ used in the previous section. We shall make brief use of the category Space of compactly generated weak Hausdorff spaces $[11$, A.1; 22, Ch. 5], "the category of spaces in which algebraic topologists customarily work" [22, p. 37]. We denote the internal function complex in $s$ Set by $\operatorname{Hom}_{s \mathbf{S e t}}(\cdot, \cdot)$ as in $[12$, I.5]. 
3.4. Theorem. Let $X$ be a smooth manifold and $Y$ be a compactly generated weak Hausdorff space. If $\mathcal{B}$ is a basis for a binoetherian subtopology on an open set $U$ in $X$, viewed as a subdiagram of the site of $X$, then $\mathcal{C}(U, Y)$ is the homotopy limit of $\mathcal{C}(\cdot, Y) \mid \mathcal{B}$.

Here, smooth means at least once continuously differentiable. For this result, the most important consequence of $X$ being a smooth manifold is that every open subset of $X$ is cofibrant since it has a triangulation $[26,10.6]$. Surely, the class of spaces with this property is much larger than the class of smooth manifolds, but I am not aware of any description of it. We also need every open subset of $X$ to be normal.

Proof. By Theorem 3.3, we need to verify that the simplicial sheaf $s \mathcal{C}(\cdot, Y)$ on $X$ satisfies excision. We will show that the simplicial presheaf $F=\operatorname{Hom}_{s \operatorname{Set}}(s \cdot, s Y)$ on $X$ is pointwise fibrant and satisfies excision and that $F$ is coarsely weakly equivalent to $s \mathcal{C}(\cdot, Y)$. Note first that $F(\varnothing)$ is the singular set of a point, so $F(\varnothing)$ is contractible.

Consider the functor $H=\mathbf{H o m}_{s \text { Set }}(\cdot, s Y)$ from $s \mathbf{S e t}^{\text {op }}$ to $s$ Set. By Quillen's Axiom SM7 for a simplicial model category [12, II.3.1], if $A \rightarrow B$ is a cofibration in $s$ Set, then the induced map $H(B) \rightarrow H(A)$ is a fibration. Hence, $F$ is flabby and pointwise fibrant. If we knew that $F$ was a sheaf, the proof that $F$ satisfies excision would end here, but we do not. The internal function space in $\operatorname{Space}$ is $k \mathcal{C}(\cdot, \cdot)$, where $k$ is the $k$-ification functor from the category of topological spaces to Space and $\mathcal{C}(\cdot, \cdot)$ carries the compact-open topology. By adjunction (at the level of simplicial categories), $H=\operatorname{skC}(|\cdot|, Y)[12$, II.3.14], but $s k=s$, so $H=s \mathcal{C}(|\cdot|, Y)$. If $A \rightarrow B$ is a weak equivalence of simplicial sets, then the weak equivalence $|A| \rightarrow|B|$ has a homotopy inverse, which induces a homotopy inverse for $k \mathcal{C}(|B|, Y) \rightarrow k \mathcal{C}(|A|, Y)$, so $H(B) \rightarrow H(A)$ is a weak equivalence. Finally, since $H$ has a left adjoint [12, II.2] (this is one of the defining properties of a simplicial category), $H$ preserves limits, i.e., takes colimits in $s$ Set to limits in $s$ Set.

Since $H$ preserves limits and weak equivalences and turns cofibrations into fibrations, $H$ turns homotopy pushouts in $s$ Set, i.e., homotopy pullbacks in $s \mathbf{S e t}^{\mathrm{op}}$, into homotopy pullbacks. Also, the singular functor $s$ turns homotopy pushouts of cofibrant spaces into homotopy pushouts in $s$ Set. To prove that $F$ satisfies excision, it therefore suffices to show that if $U$ and $V$ are open subsets of $X$, then the square

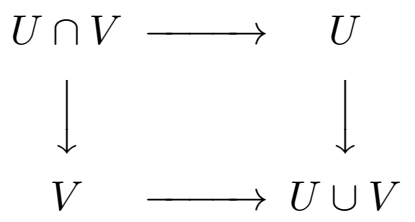

is a homotopy pushout. To calculate the homotopy pushout of $U \leftarrow U \cap V \rightarrow V$, we factor $U \cap V \hookrightarrow U$ through its mapping cylinder $M$ into a cofibration followed by a weak equivalence [22, 6.3], and take the ordinary pushout of the diagram $M \leftarrow U \cap V \rightarrow V$. The homotopy pushout $P$ turns out to be the product $(U \cap V) \times[0,1]$ with $U$ glued to $(U \cap V) \times\{0\}$, and $V$ to $(U \cap V) \times\{1\}$. We need to verify that the projection $P \rightarrow U \cup V$ is a weak equivalence. It is easy to see that a section of the projection is a homotopy inverse 
for it, and finding a section is tantamount to finding a continuous function $U \cup V \rightarrow[0,1]$ equal to 0 on $U \backslash V$ and 1 on $V \backslash U$. Since $U \cup V$ is normal, such a function is provided by the Urysohn lemma.

Finally, since every open subset $U$ of $X$ is cofibrant, the weak equivalence $|s U| \rightarrow U$ has a homotopy inverse, so the induced map $s \mathcal{C}(U, Y) \rightarrow s \mathcal{C}(|s U|, Y)=F(U)$ is a weak equivalence. Since $F$ satisfies excision, so does $s \mathcal{C}(\cdot, Y)$.

\section{Complex manifolds as simplicial sheaves on the Stein site}

Let $\mathcal{M}$ be the category of complex manifolds (second countable but not necessarily connected) and holomorphic maps. In this section, we shall embed $\mathcal{M}$ into a model category, suitable for a homotopy-theoretic interpretation of the Oka-Grauert property.

Let $\mathcal{S}$ be the category of Stein manifolds and holomorphic maps. This is a small category (or at least equivalent to one), since a connected Stein manifold can be embedded into Euclidean space. We view $\mathcal{S}$ as a site with the "usual" topology, in which a cover of a Stein manifold $S$ consists of a family of isomorphisms onto Stein open subsets of $S$ which cover $S$. We only need to verify that covers can be pulled back to covers [21, III.2]. This is implied by the following lemma.

4.1. Lemma. Let $f: X \rightarrow Y$ be a holomorphic map between complex manifolds. If $X$ is Stein and $V$ is a Stein open subset of $Y$, then the preimage $f^{-1}(V)$ is Stein.

Note that the lemma shows that the intersection of finitely many Stein open subsets of a complex manifold is Stein. (The ambient manifold need not be Stein.) Namely, for two subsets $U$ and $V$, the intersection is the preimage of $V$ under the inclusion $U \hookrightarrow U \cup V$. For more sets, iterate this.

Proof. Holomorphic functions separate points on $U=f^{-1}(V)$ since they do on $X$. To show that $U$ is holomorphically convex, we follow [15, 2.5.14]. Let $K \subset U$ be compact. We need to show that the holomorphic hull $\hat{K}_{U}$ of $K$ in $U$ is compact. Since $\hat{K}_{X}$ is compact and contains $\hat{K}_{U}$, it suffices to show that $\hat{K}_{U}$ is closed in $U$. Now $f(K) \subset V$ is compact, so $\widehat{f(K)}_{V}$ is compact. If $h \in \mathcal{O}(V)$ and $x \in \hat{K}_{U}$, then $|h(f(x))| \leq\|h \circ f\|_{K}=\|h\|_{f(K)}$, so $f(x) \in \widehat{f(K)}_{V}$. Hence, $f$ maps the closure of $\hat{K}_{U}$ into $\widehat{f(K)_{V}} \subset V$, so the closure is in $U$.

We shall refer to this topology on $\mathcal{S}$ as the fine topology and to the trivial topology, in which a cover consists of a single isomorphism, as the coarse topology. Note that a point, denoted $\mathfrak{p}$, is the final object in $\mathcal{S}$, and the empty manifold $\varnothing$ is the initial object.

Let $p$ be a point in a Stein manifold $S$. If $F$ is a presheaf on $\mathcal{S}$, we define the stalk $F_{p}$ of $F$ at $p$ to be the filtered colimit of the sets of sections $F(U)$, where $U$ is a Stein neighbourhood of $p$ in $S$. By restricting to the small site of each Stein manifold, we see that the family of stalk functors ${ }_{p}$ : Shv $\mathcal{S} \rightarrow$ Set, $F \mapsto F_{p}, p \in S \in \mathcal{S}$, is faithful, meaning that maps of sheaves are equal if they induce the same maps on all stalks. Since 
homotopy groups respect filtered colimits of simplicial sets, we see that a map $f: F \rightarrow G$ of simplicial presheaves on $\mathcal{S}$ is a fine weak equivalence (in the sense of Jardine) if and only if the induced map of stalks $f_{p}: F_{p} \rightarrow G_{p}$ is a weak equivalence for all $p \in S \in \mathcal{S}$.

Let us remark that the stalk functors just defined really are stalks (or points of $\mathcal{S}$ ) in the sense of topos theory: they have right adjoints and preserve finite limits as functors Shv $\mathcal{S} \rightarrow$ Set (so in particular, $\mathcal{S}$ has enough points). First of all, since limits of sheaves are taken pointwise and finite limits commute with filtered colimits in Set [20, IX.2], the stalk functor $\cdot p$ preserves finite limits. It also preserves colimits. A colimit of sheaves is the sheafification of the pointwise colimit. In $\mathcal{S}$, sheafification commutes with the restriction to the small site of any Stein manifold, because all covers can be realized in the manifold. Hence, preservation of colimits can be reduced to the case of a single manifold, where it holds by the standard result on topological spaces (the Stein open subsets form a basis for the usual topology). It is easily verified that the functor $\mathcal{S} \rightarrow$ Set obtained by restricting $\cdot{ }_{p}$ to representable sheaves is both filtering and continuous, so its Kan extension Shv $\mathcal{S} \rightarrow$ Set is a point in $\mathcal{S}[21$, VII.5,6], but since $\cdot p$ preserves colimits, it is its own Kan extension. The right adjoint of $\bullet_{p}$ can in fact be described explicitly [21, VII.5]: it takes a set $A$ to the "skyscraper sheaf" $X \mapsto \operatorname{hom}_{\text {Set }}\left(\mathcal{O}(\cdot, X)_{p}, A\right)$ on $\mathcal{S}$ (which does not really look like a skyscraper at all).

We will embed $\mathcal{M}$ into $s \operatorname{Shv} \mathcal{S}$. First note that by the Yoneda lemma [20, III.2], there is a full embedding of $\mathcal{M}$ into the category Pre $\mathcal{M}$ of presheaves of sets on $\mathcal{M}$, given by $X \mapsto \mathcal{O}(\cdot, X)$. It is easy to prove directly that this is still true for the smaller site $\mathcal{S}$.

4.2. Proposition. The Yoneda functor $\mathcal{M} \rightarrow \operatorname{Pre} \mathcal{S}$ is a full embedding.

Proof. We need to show that the functor is both faithful and full, i.e., that it induces bijections on all sets of morphisms. Let $f, g: X \rightarrow Y$ be maps in $\mathcal{M}$ such that $f_{*}=g_{*}$ : $\mathcal{O}(\cdot, X) \rightarrow \mathcal{O}(\cdot, Y)$. Let $\iota$ be the map $\mathfrak{p} \rightarrow X$ with image $\{p\}$ for $p \in X$. Since $f \circ \iota=g \circ \iota$, we have $f(p)=g(p)$ and $f=g$.

As for fullness, let $\alpha: \mathcal{O}(\cdot, X) \rightarrow \mathcal{O}(\cdot, Y)$ be a map in Pre $\mathcal{S}$. Now $\alpha: \mathcal{O}(\mathfrak{p}, X) \rightarrow$ $\mathcal{O}(\mathfrak{p}, Y)$ gives a map $f: X \rightarrow Y$. For a Stein manifold $S$ and $p \in S$ we have a diagram

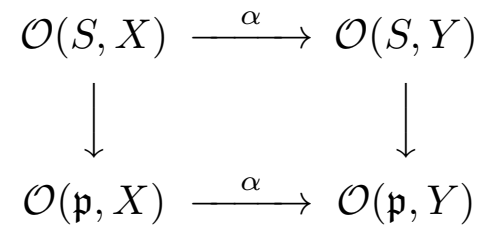

where the vertical arrows are induced by the map $\mathfrak{p} \rightarrow S$ with image $\{p\}$. Hence, for $h \in \mathcal{O}(S, X)$, we have $\alpha(h)(p)=f(h(p))$, so $\alpha=f_{*}$. Finally, $f$ is holomorphic because it preserves holomorphic maps from balls, mapping $\mathcal{O}(B, X)$ into $\mathcal{O}(B, Y)$, where $B$ is any open ball in Euclidean space.

The Yoneda embedding restricts to a full embedding of $\mathcal{M}$ into the category of presheaves of topological spaces on $\mathcal{S}$, if we equip each set $\mathcal{O}(S, X)$ with the compact-open 
topology. Finally, let us postcompose this functor with the singular functor. This yields an embedding $\mathcal{M} \rightarrow s \operatorname{Shv} \mathcal{S}$, taking a complex manifold $X$ to the simplicial presheaf $s \mathcal{O}(\cdot, X)$, which is clearly a sheaf with respect to the fine topology on $\mathcal{S}$ (recall that the singular functor preserves limits). We will not address the issue of fullness here, but only remark that every morphism $s \mathcal{O}(\cdot, X) \rightarrow s \mathcal{O}(\cdot, Y)$ is clearly given by a holomorphic map $X \rightarrow Y$ at the level of vertices. We will often view a complex manifold $X$ as an object of $s$ Shv $\mathcal{S}$ and write $X$ for $s \mathcal{O}(\cdot, X)$.

If $p$ is a point in a Stein manifold $S$ and $\operatorname{dim}_{p} S=m \geq 0$, then the stalk of a complex manifold $X$ at $p$ is simply the colimit as $n \rightarrow \infty$ of $s \mathcal{O}\left(\frac{1}{n} \mathbb{B}_{m}, X\right)$, where $\mathbb{B}_{m}$ is the open unit ball in $\mathbb{C}^{m}$ and the maps between the scaled balls $\frac{1}{n} \mathbb{B}_{m}$ are the inclusions. This is in fact a homotopy colimit, since (by the identity theorem) all the maps are cofibrations (just dualize the theory of towers in [12, VI.1]). Now for any $m \geq 0$ and $r>0, \mathcal{O}\left(r \mathbb{B}_{m}, X\right)$ is weakly equivalent to $X$ itself, so all the stalks of $X$ are weakly equivalent to $s X$.

A holomorphic map $X \rightarrow Y$ is a cofibration (with respect to either topology) if and only if it is injective. It is a coarse weak equivalence if and only if it induces a topological weak equivalence $\mathcal{O}(S, X) \rightarrow \mathcal{O}(S, Y)$ for every Stein manifold $S$. It is a fine, i.e., stalkwise, weak equivalence if and only if it is a topological weak equivalence. As for fibrations, we only remind the reader that they are defined by a right lifting property with respect to acyclic cofibrations.

Examples. Since $\mathbb{D}$ and $\mathbb{C}$ are holomorphically contractible, they are both coarsely weakly equivalent to a point. The same holds for any star-shaped domain in Euclidean space. The inclusion $\mathbb{D}^{\times} \hookrightarrow \mathbb{C}^{\times}$is a fine but not a coarse weak equivalence. Namely, by Liouville's theorem, $\mathcal{O}\left(\mathbb{C}^{\times}, \mathbb{D}^{\times}\right)=\mathbb{D}^{\times}$, but $\mathcal{O}\left(\mathbb{C}^{\times}, \mathbb{C}^{\times}\right)$has infinitely many connected components, one for each winding number about the origin.

Now let $X$ be a complex manifold. The inclusion $s \mathcal{O}(\cdot, X) \hookrightarrow s \mathcal{C}(\cdot, X)$ is a fine weak equivalence of simplicial sheaves on $\mathcal{S}$ because every cover has a refinement consisting of sets at which the inclusion is a weak equivalence: take a refinement by balls, for instance. The Oka-Grauert property is satisfied by $X$ if and only if this inclusion is a coarse weak equivalence. Theorem 2.1 states that this is equivalent to the simplicial sheaf $s \mathcal{O}(\cdot, X)$ satisfying finite excision in the sense of Section 2, meaning that for every finite cover $\left\{U_{1}, \ldots, U_{m}\right\}$ in $\mathcal{S}, s \mathcal{O}\left(\cup U_{i}, X\right)$ is not only the limit but also the homotopy limit of the diagram whose objects are the simplicial sets $s \mathcal{O}\left(U_{i_{1}} \cap \cdots \cap U_{i_{k}}, X\right)$ for $1 \leq i_{1}<\cdots<i_{k} \leq m$ and whose arrows are induced by restriction maps. This property may also be expressed by saying that $s \mathcal{O}(\cdot, X)$ is a finite homotopy sheaf. We can now state Gromov's Oka principle and our interpretation of its conclusion, the Oka-Grauert property, as follows.

4.3. Theorem. Let $X$ be a complex manifold.

(1) The fine weak equivalence $s \mathcal{O}(\cdot, X) \hookrightarrow s \mathcal{C}(\cdot, X)$ of simplicial sheaves on the Stein site is coarsely acyclic if and only if $s \mathcal{O}(\cdot, X)$ is a finite homotopy sheaf.

(2) If $X$ has a spray, then $X$ represents a finite homotopy sheaf on the Stein site. 
Finite excision for pointwise fibrant simplicial presheaves on $\mathcal{S}$ is clearly invariant under coarse weak equivalences. Let us show that descent implies finite excision. As remarked before Theorem 3.1, descent implies two-set excision, but since we are not working on a topological space (the union of Stein open subsets is usually not Stein), we cannot simply refer to Theorem 3.3 to get finite excision. A simplicial presheaf satisfying descent is coarsely weakly equivalent to a finely fibrant simplicial sheaf $F$ on $\mathcal{S}$, so it suffices to show that $F$ satisfies finite excision. Let $S$ be a Stein manifold. Via its étale space, $F \mid S$ extends to a simplicial sheaf $\tilde{F}$ on $S$ with its usual topology. It suffices to show that $\tilde{F}$ is flabby; we then invoke Theorem 3.3. By the Yoneda lemma, if $X$ is a Stein manifold, then $F(X)=\operatorname{Hom}_{s} \operatorname{Shv} \mathcal{S}(\hat{\mathcal{O}}(\cdot, X), F)$, where the sheaf $\mathcal{O}(\cdot, X)=\operatorname{hom}_{\mathcal{S}}(\cdot, X)$ of sets on $\mathcal{S}$ has been turned into a simplicial sheaf $\hat{\mathcal{O}}(\cdot, X)$ in the trivial way (the same set in all degrees; all face and degeneracy maps are the identity). If $V$ is an open subset of $S$, let $\mathcal{B}$ be a Stein basis for the topology of $V$, viewed as a subdiagram of the site of $S$. Then $\mathcal{O}(\cdot, V)$ is the sheaf colimit of the diagram $\mathcal{O}(\cdot, \mathcal{B})$, and

$$
\operatorname{Hom}_{s \operatorname{Shv}} \mathcal{S}(\hat{\mathcal{O}}(\cdot, V), F)=\lim \operatorname{Hom}_{s} \operatorname{Shv} \mathcal{S}(\hat{\mathcal{O}}(\cdot, \mathcal{B}), F)=\lim F(\mathcal{B})=\tilde{F}(V)
$$

If $W \subset V$ are open subsets of $S$, then the induced map $\hat{\mathcal{O}}(\cdot, W) \rightarrow \hat{\mathcal{O}}(\cdot, V)$ is clearly a cofibration (pointwise injection), so by Quillen's Axiom SM7, the restriction map $\tilde{F}(V) \rightarrow$ $\tilde{F}(W)$ is a fibration, and the proof is complete.

Note, finally, that a nondiscrete complex manifold $X$ is never flabby, let alone coarsely or finely fibrant. Namely, let $D_{r}$ be the open disc of radius $r$ centred at the origin in the complex plane. The inclusion $D_{1} \hookrightarrow D_{2}$ is a monomorphism in $\mathcal{S}$, but since there are holomorphic maps $D_{1} \rightarrow X$ that do not extend holomorphically to $D_{2}$, it is easily seen that the restriction map $s \mathcal{O}\left(D_{2}, X\right) \rightarrow s \mathcal{O}\left(D_{1}, X\right)$ is not a fibration.

\section{Partial descent on the quasi-projective site}

It is natural to ask whether a finite homotopy sheaf on $\mathcal{S}$ satisfies descent. This would turn Gromov's Oka principle into a descent theorem, somewhat analogous to such results as Brown-Gersten descent and (unstable) Nisnevich descent in algebraic geometry. I do not know the answer: the finiteness properties that make descent possible in algebra — the Zariski topology being binoetherian, essentially — do not hold in analysis. We hope to address this question in future work. In the meantime, let us show how BrownGersten descent easily implies partial descent of sorts for quasi-projective manifolds with the Oka-Grauert property.

Let $\mathcal{A}$ be the category of quasi-projective complex manifolds, i.e., smooth Zariski open sets in projective varieties, and algebraic maps. We put the usual Zariski topology on $\mathcal{A}$ by defining a cover of a quasi-projective manifold $X$ to be a family of isomorphisms onto Zariski open subsets of $X$ which cover $X$ (covers can be pulled back to covers because algebraic maps are Zariski continuous). Taking $X$ to the sheaf of $\operatorname{sets} \mathcal{O}(\cdot, X)$ on the 
small site $\mathcal{A}$ defines an embedding of $\mathcal{A}$ into $\operatorname{Shv} \mathcal{A}$ (it is faithful because the Yoneda embedding $X \mapsto \operatorname{hom}_{\mathcal{A}}(\cdot, X)$ is). As before, we equip each set of holomorphic maps with the compact-open topology, apply the singular functor, and obtain an embedding of $\mathcal{A}$ into the model category $s \operatorname{Shv} \mathcal{A}$ of simplicial sheaves on $\mathcal{A}$, taking a quasi-projective manifold $X$ to the simplicial sheaf $s \mathcal{O}(\cdot, X)$. This embedding is not full, but every morphism $s \mathcal{O}(\cdot, X) \rightarrow s \mathcal{O}(\cdot, Y)$ is given by a holomorphic map $X \rightarrow Y$ at the level of vertices.

Now let $X$ be a quasi-projective manifold. We claim that the simplicial sheaf $G=$ $s \mathcal{C}(\cdot, X)$ on $\mathcal{A}$ satisfies descent. Let $G \rightarrow F$ be a fibrant model for $G$, i.e., an acyclic cofibration to a fibrant simplicial sheaf $F$ on $\mathcal{A}$. Let $A$ be a quasi-projective manifold with the Zariski topology. By Theorem 3.4, $G \mid A$ satisfies excision. So does $F \mid A$, since it is flabby. Hence, the weak equivalence $G \rightarrow F$ is pointwise acyclic by Proposition 3.2, and $G$ satisfies descent.

Suppose now that $X$ has the Oka-Grauert property, so $s \mathcal{O}(S, X) \hookrightarrow G(S)$ is a weak equivalence for every Stein manifold $S$. Every cover in $\mathcal{A}$ has a refinement consisting of Stein Zariski open sets, so $s \mathcal{O}(\cdot, X) \rightarrow G$ is an acyclic cofibration between simplicial sheaves on $\mathcal{A}$. Hence, the composition $s \mathcal{O}(\cdot, X) \rightarrow F$ is a fibrant model for $s \mathcal{O}(\cdot, X)$, and $s \mathcal{O}(S, X) \rightarrow F(S)$ is a weak equivalence for every Stein manifold $S$ in $\mathcal{A}$. Since any two fibrant models for the same object in $s \operatorname{Shv} \mathcal{A}$ are pointwise weakly equivalent, this holds for every fibrant model for $s \mathcal{O}(\cdot, X)$, and we have proved the following "Stein descent theorem".

5.1. Theorem. Let $X$ be a quasi-projective manifold and $F$ be a fibrant model for $s \mathcal{O}(\cdot, X)$ in $s \mathrm{Shv} \mathcal{A}$. If $X$ has the Oka-Grauert property, then $s \mathcal{O}(S, X) \rightarrow F(S)$ is a weak equivalence for every quasi-projective Stein manifold $S$.

\section{REFERENCES}

1. K. S. Brown and S. M. Gersten, Algebraic K-theory as generalized sheaf cohomology, Algebraic Ktheory, I: Higher K-theories (Proc. Conf., Battelle Memorial Inst., Seattle, Wash., 1972), Lecture Notes in Math. 341, Springer Verlag, 1973, pp. 266-292.

2. W. G. Dwyer and J. Spaliński, Homotopy theories and model categories, Handbook of algebraic topology, North-Holland, 1995, pp. 73-126.

3. Y. Eliashberg and M. Gromov, Embeddings of Stein manifolds of dimension $n$ into the affine space of dimension $3 n / 2+1$, Ann. of Math. 136 (1992), 123-135.

4. F. Forstnerič, On complete intersections, Ann. Inst. Fourier (Grenoble) 51 (2001), 497-512.

5. The Oka principle, lifting of holomorphic maps and removability of intersections, preprint, arXiv:math.CV/0101238.

6. _ The Oka principle for miltivalued sections of ramified mappings, preprint, arXiv:math.CV/0107039.

7. The Oka principle for sections of subelliptic submersions, preprint, arXiv:math.CV/0110201.

8. F. Forstnerič and J. Prezelj, Oka's principle for holomorphic fiber bundles with sprays, Math. Ann. 317 (2000), 117-154.

9. __ Extending holomorphic sections from complex subvarieties, Math. Z. 236 (2001), 43-68.

10. __ Oka's principle for holomorphic submersions with sprays, Math. Ann. 322 (2002), 633-666. 
11. R. Fritsch and R. A. Piccinini, Cellular structures in topology, Cambridge Studies in Advanced Math. 19, Cambridge University Press, 1990.

12. P. G. Goerss and J. F. Jardine, Simplicial homotopy theory, Progress in Mathematics 174, Birkhäuser Verlag, 1999.

13. M. Gromov, Oka's principle for holomorphic sections of elliptic bundles, Jour. Amer. Math. Soc. 2 (1989), 851-897.

14. P. S. Hirschhorn, Localization of model categories, available at http://math.mit.edu/ psh (2001).

15. L. Hörmander, An introduction to complex analysis in several variables, North-Holland Publ. Co., 1973.

16. M. Hovey, Model categories, Math. Surveys and Monographs 63, Amer. Math. Soc., 1999.

17. J. F. Jardine, Simplicial presheaves, Jour. Pure Appl. Algebra 47 (1987), 35-87.

18. - Boolean localization, in practice, Doc. Math. 1 (1996), 245-275.

19. J. Leiterer, Holomorphic vector bundles and the Oka-Grauert principle, Several Complex Variables IV, Encyclopaedia of Mathematical Sciences vol. 10, Springer-Verlag, 1990, pp. 63-103.

20. S. Mac Lane, Categories for the working mathematician, Graduate Texts in Mathematics 5, SpringerVerlag, 1971.

21. S. Mac Lane and I. Moerdijk, Sheaves in geometry and logic: a first introduction to topos theory, Springer-Verlag, 1992.

22. J. P. May, A concise course in algebraic topology, Chicago Lectures in Mathematics, The University of Chicago Press, 1999.

23. J. Milnor, Singular points of complex hypersurfaces, Annals of Math. Studies 61, Princeton University Press, 1968.

24. S. A. Mitchell, Hypercohomology spectra and Thomason's descent theorem, Algebraic K-theory (Toronto, ON, 1996), Fields Institute Communications 16, American Math. Society, 1997.

25. F. Morel and V. Voevodsky, $A^{1}$-homotopy theory of schemes, Inst. Hautes Études Sci. Publ. Math. 90 (1999), 45-143.

26. J. R. Munkres, Elementary differential topology, Annals of Math. Studies 54, revised edition, Princeton University Press, 1966.

27. D. G. Quillen, Homotopical algebra, Lecture Notes in Mathematics 43, Springer-Verlag, 1967.

28. J. Schürmann, Embeddings of Stein spaces into affine spaces of minimal dimension, Math. Ann. 307 (1997), 381-399.

29. Y.-T. Siu, Every Stein subvariety admits a Stein neighborhood, Invent. Math. 38 (1976), 89-100.

30. E. M. Stein, Singular integrals and differentiability properties of functions, Princeton Univ. Press, 1970.

31. V. Voevodsky, $\mathbf{A}^{1}$-homotopy theory, Proceedings of the International Congress of Mathematicians, vol. I (Berlin, 1998), Doc. Math., extra vol. I (1998), 579-604.

32. — Voevodsky's Seattle lectures: K-theory and motivic cohomology, Algebraic K-theory (Seattle, WA, 1997), Proc. Symp. Pure Math. 67, Amer. Math. Soc., 1999.

Department of Mathematics, University of Western Ontario, London, Ontario N6A 5B7, CANADA

E-mail address: larusson@uwo.ca 